\title{
Ethanol Steam Reforming for Hydrogen Production Over Hierarchical Macroporous Mesoporous SBA-15 Supported Nickel Nanoparticles
}

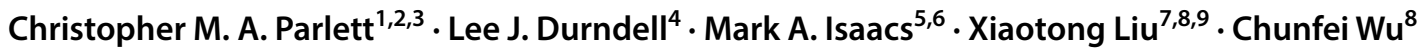

Published online: 6 May 2020

(c) The Author(s) 2020

\begin{abstract}
The influence of complementary macropores, present in hierarchical macroporous mesoporous SBA-15, on the performance of supported Ni nanoparticles for ethanol steam reforming has been investigated. The increased open nature of the architecture, afforded through the incorporation of the secondary macropore network, enables superior metal dispersion. This, in turn, enhances catalytic hydrogen production performance through the generation of a greater density of active sites.
\end{abstract}

Keywords Hierarchical porous materials $\cdot$ Macroporous mesoporous SBA-15 $\cdot$ Hydrogen production $\cdot$ Ethanol steam reforming $\cdot$ Nickel $\cdot$ Nanoparticles

\section{Introduction}

Hydrogen represents a clean and potentially sustainable fuel, which can address increasing uncertainties regarding the availability and accessibility of fossil fuel reserves.

Christopher M. A. Parlett

christopher.parlett@manchester.ac.uk

1 Department of Chemical Engineering and Analytical Science, University of Manchester, Manchester M13 9PL, UK

2 Diamond Light Source, Harwell Science and Innovation Campus, Didcot OX11 0DE, Oxfordshire, UK

3 The University of Manchester at Harwell, Diamond Light Source, Harwell Science and Innovation Campus, Didcot OX11 0DE, Oxfordshire, UK

4 School of Geography, Earth and Environmental Sciences, University of Plymouth, Plymouth PL4 8AA, UK

5 HarwellXPS, Research Complex at Harwell, Harwell Science and Innovation Campus, Didcot OX11 0FA, Oxfordshire, UK

6 Department of Chemistry, University College London, London WC1H 0AJ, UK

7 School of Engineering and Computer Science, Faculty of Science and Engineering, University of Hull, Hull HU6 7RX, UK

8 School of Chemistry and Chemical Engineering, Queen's University Belfast, Belfast BT7 1NN, Northern Ireland, UK

9 Vision Academy, 398 Jiangsu Street, Shanghai 200000, China
Furthermore, a hydrogen-based energy economy could address concerns regarding the impact of fossil fuel combustion on climate change, which itself is driving such research into alternative fuel feedstocks [1]. As such, the development of alternative environmental friendly renewable fuels is vital to meeting legally mandated targets for $\mathrm{EU} \mathrm{CO}_{2}$ emissions [2]. Biomass has been widely accepted as a sustainable chemical feedstock, with hydrogen derived from catalytic bioethanol steam reforming acknowledged to be a viable production route [3]. The development of novel catalytic systems, with optimal on-stream performance and stability, is, therefore, a key parameter to enabling this future process for sustainable fuel production.

Low-cost-earth-abundant base metals, such as $\mathrm{Ni}$, are commonly the active site of choice for ethanol steam reforming (ESR) due to superior economics [4], but routinely suffer on-stream deactivation by coking and sintering $[5,6]$. Fine-tuning of reaction conditions can inhibit carbon deposition, through increased process temperature or addition of $\mathrm{O}_{2}$ to drive coke oxidation, but at the expense of increased potential for active site sintering, operating costs, or active site oxidation [7]. Alternatively, modifying the chemical nature of the support structure provides a second strategy to address coke accumulation. Alkali metal or alkali earth metal promotors switch-off ethanol dehydration and ethylene polymerisation, through tuning of the acid/base nature [7], in contrast acidic supports, such as zeolites, enhance ethanol dehydration the formation of ethylene increase coking and deactivation [6]. Basic oxides of $\mathrm{Ca}$ and $\mathrm{Mg}$ have been 
used to increase $\mathrm{H}_{2}$ production performance via sorptionenhance steam reforming [8,9], praseodymium and barium oxides have also promoted enhance steam reforming activity through reduced Ni sintering $[10,11]$, whereas rare earth and transition metal oxides, e.g. $\mathrm{CeO}_{2}$ or $\mathrm{ZrO}_{2}$, impart labile surface oxygen which assists oxidation of carbon deposits to $\mathrm{CO}_{2}$ [12]. The ratio of these to the active phase playing an important role in Ni crystallite size, which in turn controls activity and resistance to carbon deposition [13]. Such reducible oxide materials typically exhibit low intrinsic surface areas, and as such are ineffective for stabilising highly dispersed base metal active sites, whilst porous analogues typically suffer reduced hydrothermal stability. Alternative approaches have been to co-deposit these oxides in conjunction with the active Ni species [14-16], yet these could result in a capping layer on top of the active species, whereas adlayer oxide growth has also shown potential to address the inherent issues of these oxides [17].

We have previously demonstrated the capacity of silica as a support material in ESR, with its intrinsic high surface area and chemically inertness, for both commercial and simple sol-gel synthesised silica, enabling high active site dispersion [18], and accessibility [19]. These beneficial attributes can be further enhanced through the utilisation of ordered mesoporous silicas, such as SBA-15, SBA-16 and KIT-6, which have demonstrated superior active site generation whilst overcoming internal mass diffusion constraints for $\mathrm{C} 4$ and $\mathrm{C} 9$ alcohols [20]. Controlling ESR performance over $\mathrm{Ni}$ deposited on SBA-15 is dependent on the location of the active species. Sintering and coking are apparent where the active site exists as large nanoparticles located on the external surfaces of the support skeleton, with nanoparticle size $>$ mesopore diameter [15]. Whilst promotors (including $\mathrm{Ce}, \mathrm{Au}, \mathrm{Ca}$, or $\mathrm{Mg}[14,21,22])$ afford one strategy to tackle this undesirable phenomenon, encapsulating the $\mathrm{Ni}$ active species provides an alternative, for example, successfully incorporating the Ni sites within the mesopores of SBA-15 or KIT-6 [23]. Control of the location of the Ni species in this way prohibits sintering, via confinement, with the more open 3-dimensional architecture of KIT-6 also mitigating against pore blockage through coking, resulting in high onstream stability. The incorporation of ethylene glycol within the impregnation protocol being critical, similarly, EDTA has recently been shown to assist in crystallite size control [24].

The incorporation of a complementary macropore network within SBA-15, to yield macroporous mesoporous SBA-15 (MM-SBA-15), has shown to be beneficial for both active site dispersion and internal mass diffusion of bulk substrates for liquid-phase catalytic selective oxidation reactions [25]. Here we further demonstrate the benefit of this secondary structural framework within MM-SBA-15 for the gas-phase catalytic ESR over deposited Ni nanoparticles.

\section{Experimental}

MM-SBA-15, comprising a framework of $400 \mathrm{~nm}$ spherical macropores with a two-dimensional hexagonal close-packed assembly of mesopore channels $(P 6 \mathrm{~mm})$, was synthesised by the method of Dhainaut et al. [26] MM-SBA-15 was doped with Ni nanoparticles, via a glycol assisted impregnation to enhance metal dispersion, and screened for catalytic hydrogen production from ESR [23].

\section{1 $400 \mathrm{~nm}$ Polystyrene Nanosphere Synthesis}

Styrene $\left(50 \mathrm{~cm}^{3}\right)$ and divinylbenzene $\left(9.5 \mathrm{~cm}^{3}\right)$ were each washed three times with sodium hydroxide solution $(0.1 \mathrm{M}$, $1: 1 \mathrm{vol} / \mathrm{vol})$ followed by three washings with deionised water $(1: 1 \mathrm{vol} / \mathrm{vol})$ to remove the polymerisation inhibitors. The washed organic phases were added to degassed deionised water $\left(377 \mathrm{~cm}^{3}\right)$, purged under $\mathrm{N}_{2}\left(10 \mathrm{~cm}^{3} \mathrm{~min}^{-1}\right)$, at $70{ }^{\circ} \mathrm{C}$. An aqueous initiator solution of potassium persulfate $(0.16 \mathrm{~g})$ in deionised water $\left(12 \mathrm{~cm}^{3}\right)$ was added dropwise, and the resulting mixture stirred at $300 \mathrm{rpm}$ under $\mathrm{N}_{2}(10$ $\mathrm{cm}^{3} \mathrm{~min}^{-1}$ ) for $18 \mathrm{~h}$. The solid polystyrene nanospheres were isolated by filtration and washed with deionised water.

\subsection{MM-SBA-15 Synthesis}

Pluronic P123 (10 g) was dissolved in deionised water (75 $\left.\mathrm{cm}^{3}\right)$ and hydrochloric acid $\left(2 \mathrm{M}, 290 \mathrm{~cm}^{3}\right)$ with stirring at $35{ }^{\circ} \mathrm{C}$. Polystyrene beads ( $45 \mathrm{~g}$ ) were added to the solution and left to stir for $1 \mathrm{~h}$. Tetraethoxysilane $\left(22.8 \mathrm{~cm}^{3}\right)$ was added and left for $20 \mathrm{~h}$ with agitation at $500 \mathrm{rpm}$. The resulting sol-gel was aged within a sealed container for $24 \mathrm{~h}$ at $80^{\circ} \mathrm{C}$ under static conditions. The solid was filtered, washed with deionised water $\left(1000 \mathrm{~cm}^{3}\right)$ and dried at room temp before calcination at $550{ }^{\circ} \mathrm{C}$ for $6 \mathrm{~h}$ in air (ramp rate $\left.0.5^{\circ} \mathrm{C} \mathrm{min}^{-1}\right)$.

\subsection{Ethylene Glycol Assisted Ni Impregnation}

MM-SBA-15 (1.5 g) was stirred in aqueous impregnation solution $(12 \mathrm{ml})$ containing nickel nitrate hexahydrate to give metal loadings of 10,5 and 2.5 and $1 \mathrm{wt} \%$ and ethylene glycol (1:1 Ni:glycol mol ratio) for $16 \mathrm{~h}$. The solution was heated to $50{ }^{\circ} \mathrm{C}$ and stirred for $8 \mathrm{~h}$ to form a dry powder. The resulting solid was calcined at $400{ }^{\circ} \mathrm{C}$ for $2 \mathrm{~h}$ in air (ramp rate $1{ }^{\circ} \mathrm{C} \mathrm{min}^{-1}$ ) followed by reduction at $600{ }^{\circ} \mathrm{C}$ for $2 \mathrm{~h}$ under flowing hydrogen (ramp rate $1{ }^{\circ} \mathrm{C} \mathrm{min}^{-1}$ hydrogen flow $10 \mathrm{~cm}^{3} \mathrm{~min}^{-1}$ ). 


\subsection{Characterisation}

Powder X-ray diffraction (XRD) patterns were collected on a Bruker D8 Advance Diffractometer with a LynxEye high-speed strip detector using $\mathrm{Cu} \mathrm{K} \alpha(1.54 \AA)$ radiation, $\mathrm{Si}(111)$ monochromated, Ni filter and calibrated to quartz. Low-angle data were collected between $2 \theta=0.45^{\circ}-8^{\circ}$ and wide-angle data between $2 \theta=10^{\circ}-80^{\circ}$. Nitrogen adsorption-desorption isotherms were recorded on a Quantachrome Autosorb IQTPX porosimeter with analysis using ASiQwin v3.01 software. Samples were degassed at $150^{\circ} \mathrm{C}$ for $12 \mathrm{~h}$ prior to recording $\mathrm{N}_{2}$ adsorption/desorption isotherms. BET surface areas were calculated over the relative pressure range $0.02-0.2$ with microporosity assessed by the $\mathrm{t}$-plot method over the range $\mathrm{P} / \mathrm{P} 0=0.2-0.5$. Mesopore properties were calculated by the fitting of isotherms to the relevant DFT kernel within the software package. XPS analysis was performed using a Kratos Axis SUPRA XPS fitted with a monochromated $\mathrm{Al} \mathrm{K} \alpha \mathrm{X}$-ray source $(1486.7 \mathrm{eV})$. Survey scans were recorded at a pass energy of $160 \mathrm{eV}$, and high-resolution scans recorded at a pass energy of $20 \mathrm{eV}$. Electronic charge neutralization was achieved using a magnetic immersion lens. All sample data was recorded at a pressure below $10^{-8}$ Torr and a room temperature of $294 \mathrm{~K}$. Data was analysed using CasaXPS v2.3.19PR1.0. High resolution scanning transmission microscopy (STEM) images were obtained on an aberration-corrected JEOL 2100-F microscope operated at $200 \mathrm{kV}$, with image analysis using ImageJ 1.41 software. Samples were dispersed in methanol and drop cast on 200-mesh carbon coated copper grids and dried under ambient conditions.

\subsection{Catalytic Screening}

Ethanol steam reforming for hydrogen production was carried out using a two-stage fixed-bed reaction system. The two stages were heated externally using two heaters (each one has a diameter of $25 \mathrm{~mm}$ and length of $150 \mathrm{~mm}$ ) and con-

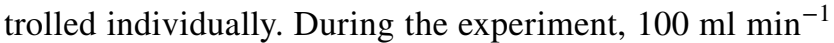
$\mathrm{N}_{2}$ gas (controlled by a mass flow meter) was used as the carrier gas. $0.2 \mathrm{~g}$ catalyst was placed into the second reactor and heated by the second furnace to $600{ }^{\circ} \mathrm{C}$, while the first furnace (quartz wool was placed inside for enhancing the dispersion of vapours) was heated to $200{ }^{\circ} \mathrm{C}$. The gas hourly space velocity was $6000 \mathrm{~h}^{-1}$.

When temperatures stabilized, a mixture of ethanol and water (molar ratio 1:4, total flow rate of $3 \mathrm{ml} \mathrm{h}^{-1}$ ) was injected using a syringe pump into the first reactor to be vaporised. The vapours passed through the second reactor for catalytic reforming reactions. The products produced from the second reactor were cooled using a condensation system (air and dry ice cooled) to collect the liquid products. The non-condensable products $\left(\mathrm{H}_{2}, \mathrm{CO}\right.$ and $\left.\mathrm{CO}_{2}\right)$ were analysed on-lined by an ETG Syngas Analyser MCA 100. Experiments were repeated in triplicate to confirm catalytic performance reproducibility. Initial and steady-state hydrogen productivity was calculated from the average hydrogen production over $0-0.16$ and $0.5-1.5 \mathrm{~h}$ respectively.

To confirm that we were not operating under reagent limiting conditions, full or equilibrium limited conversion [27], simulations to evaluate reaction equilibrium were determined using Aspen Plus ${ }^{\circledR}$. An Idea model was used, for which a Gibbs reactor was employed at $600{ }^{\circ} \mathrm{C}$ and 1 atmosphere with a mixture of ethanol and water (molar ratio of 1:4) at a flow rate of $3 \mathrm{~g} \mathrm{~h}^{-1}$, with possible products consistent with the literature [28].

\section{Results and Discussion}

The physicochemical properties of the Ni-MM-SBA-15 catalysts series were evaluated by XRD and nitrogen adsorption-desorption isotherms, shown in Fig. 1. The d10 reflection in the low-angle XRD pattern (Fig. 1a) indicates a $P 6 \mathrm{~mm}$ ordered mesopore structure, with a constant cell parameter of $\sim 9.3 \mathrm{~nm}$ irrespective of Ni loading, which agrees with our previous studies [25, 26]. Wide-angle XRD (Fig. 1b) revealed the presence of both $\mathrm{Ni}$ and $\mathrm{NiO}$ phases, with $\mathrm{Ni}[111]$ reflection at $43.9^{\circ}$ and $\mathrm{NiO}[111]$ at $36.9^{\circ}$, witnessed for loadings great than $2.5 \mathrm{wt} \%$. Unfortunately, the presence of both phases prevents Scherrer average particle size evaluation due to the overlap of the $\mathrm{NiO}[200]$ and $\mathrm{Ni}[111]$ reflections. The presence of $\mathrm{NiO}$ is attributed to the storage conditions, since the catalysts were stored in air after reduction, and was also apparent for similarly prepared $\mathrm{Ni} /$ SBA-15 series [23]. Furthermore, this oxidation indicates that the ethylene glycol was fully removed during the synthesis's thermal processing steps [29]. Nitrogen adsorption isotherms, in Fig. 1c, reveal all catalytic materials exhibit type 4 isotherms with $\mathrm{H} 1$ hysteresis, which is typical of mesoporous materials comprising uniform diameter channel pores. Brunauer-Emmett-Teller (BET) surface areas showed an inverse correlation with metal loading, spanning from $420 \mathrm{~m}^{2} \mathrm{~g}^{-1}$ to $330 \mathrm{~m}^{2} \mathrm{~g}^{-1}$ for 1 and $10 \mathrm{wt} \% \mathrm{Ni}$ loading respectively. These represent a maximum loss of $31 \%$ relative to the unfunctionalized parent, which has a surface area of $478 \mathrm{~m}^{2} \mathrm{~g}^{-1}$, whereas intermediate loadings exhibit surface areas of $392(2.5 \mathrm{wt} \%)$ and $363(5 \mathrm{wt} \%) \mathrm{m}^{2} \mathrm{~g}^{-1}$ respectively. T-plot analysis, to deconvolute the contribution of microporosity to the total surface area, confirmed that the BET surface areas are contributed to solely from mesoporosity and macroporosity, with the complementary microporosity present in conventional cooperative self-assemble synthesized mesoporous SBA-15 absent [30, 31]. Average mesopore diameters of $5.6 \mathrm{~nm}$ across the series are consistent with 
(a)

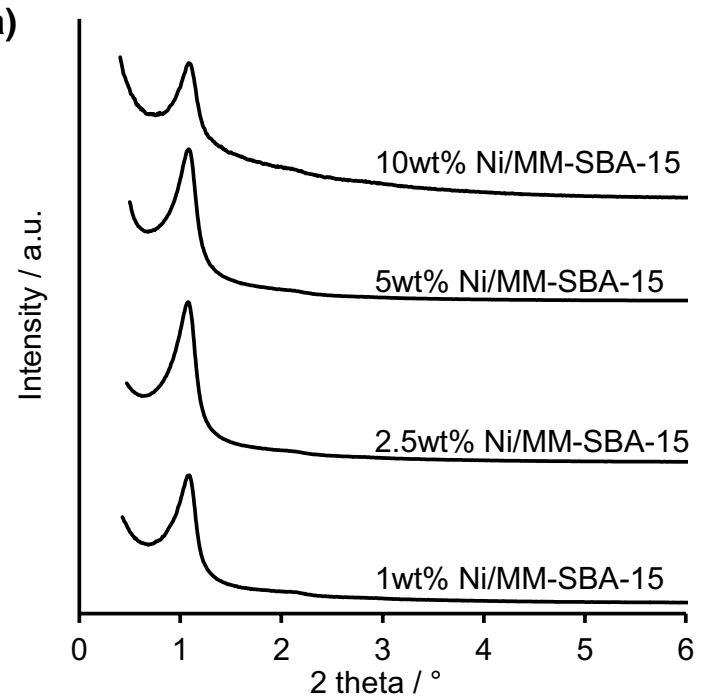

(c)

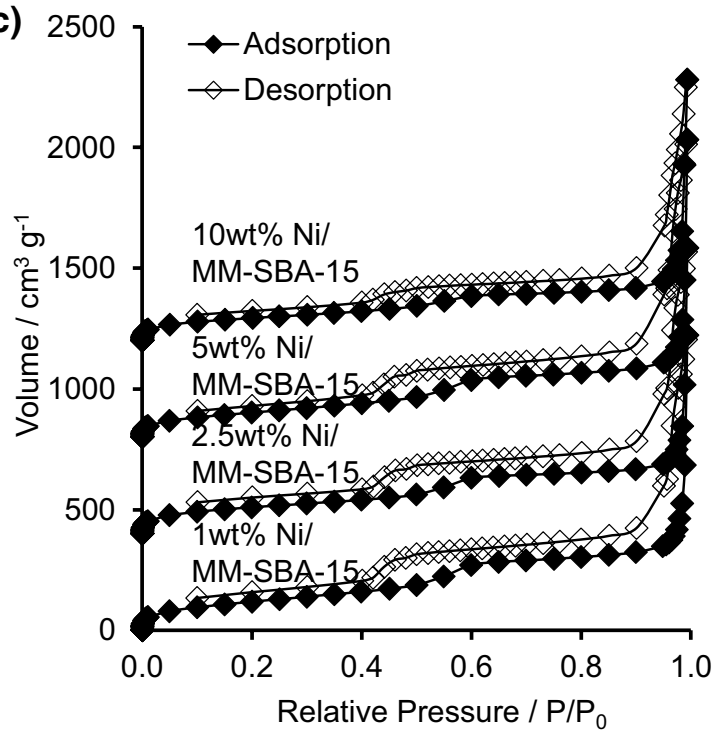

Fig. 1 Characterisation of Ni/MM-SBA-15 catalysts series; a stacked low angle powder XRD offset for clarity, $\mathbf{b}$ stacked wide angle powder XRD offset for clarity, $\mathbf{c}$ stacked nitrogen adsorption-desorption

the literature and exhibit monomodal pore size distributions, shown in Fig. 1d.

XPS was utilised to further investigate the chemical nature of the $\mathrm{Ni}$ active sites, shown in Fig. 2. As observed previously for purely mesoporous silica supports, the $\mathrm{Ni}$ species are predominately present as $\mathrm{Ni}^{(\mathrm{II})}$, apparent from binding energy peak positions which are consistent with $\mathrm{Ni}$ $2 \mathrm{p}_{3 / 2} \mathrm{NiO}$ at $856 \mathrm{eV}$. Although all samples are subjected to a reduction step during $\mathrm{Ni}$ impregnation, storage in air at room temperature leads to significant nanoparticle oxidation.

Bright field STEM confirmed the presence of both macropores and mesopores within the support framework (Fig. 3), with the macropores acting to break up the mesopore domains, thus, decreasing the mesopore
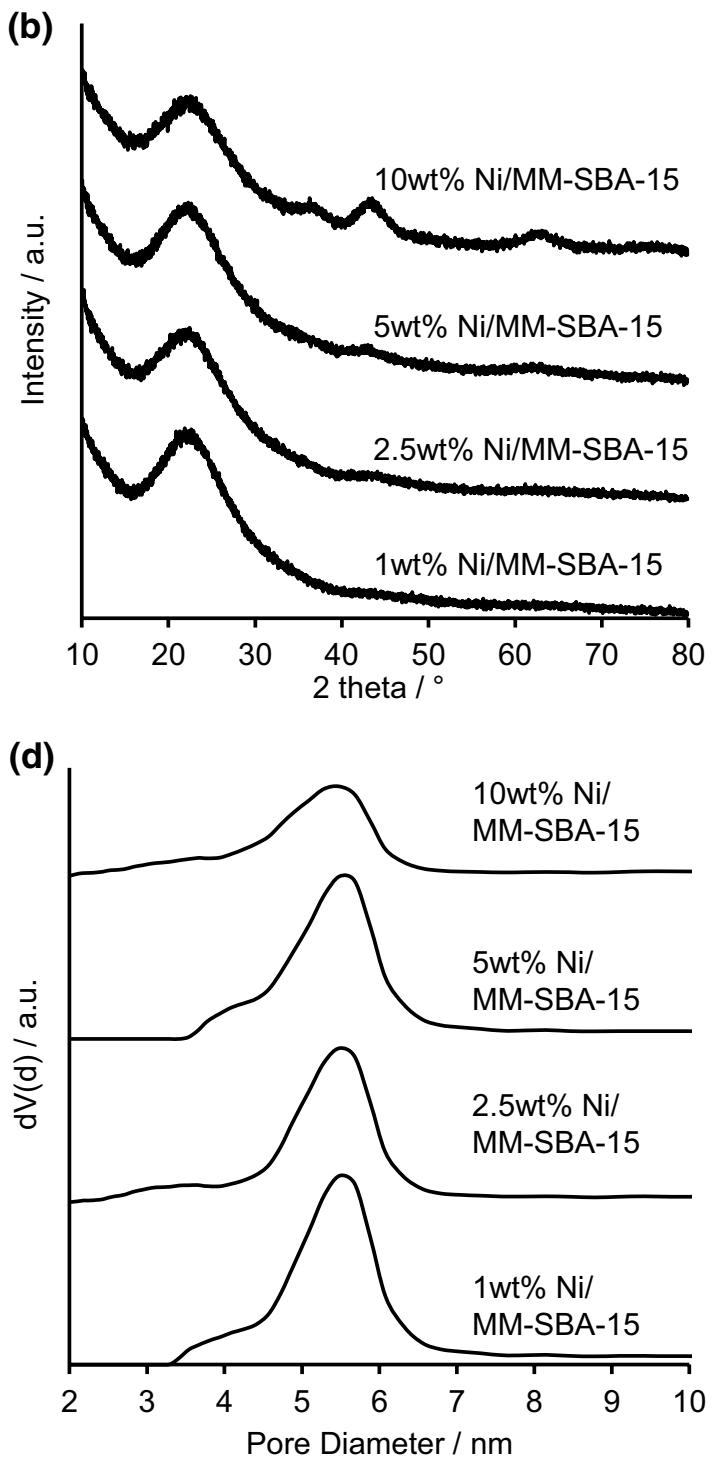

isotherms offset by 400 (2.5 wt\%), 800 (5 wt\%) and $1200(10 \mathrm{wt} \%)$ $\mathrm{cm}^{3} \mathrm{~g}^{-1}$, and $\mathbf{d}$ stacked mesopore size distribution plot offset for clarity

channel length relative to conventional SBA-15 [32]. Average macropore diameters of $360 \mathrm{~nm}$ represent only a minor contraction, during calcination, of the hard template size of $390 \mathrm{~nm}$, shown in Fig. 3b. This slight decrease is consistent with our previous investigations, with a decrease typically around $10 \%$ [33]. Figure 2c, d clearly reveal the hexagonal close packed arrangement of cylindrical channels of the mesostructures, as expected for SBA-15 [34], confirming the P6mm space group assigned by low angle XRD.

The open pore architecture afforded by MM-SBA-15 results in exceptional $\mathrm{Ni}$ dispersions, with $\mathrm{Ni}$ nanoparticles deposited throughout the material. High angle annular dark field STEM (Fig. 4), reveals the Ni nanoparticles as the brightest features due to the $\mathrm{Z}$-contrasting nature of the 


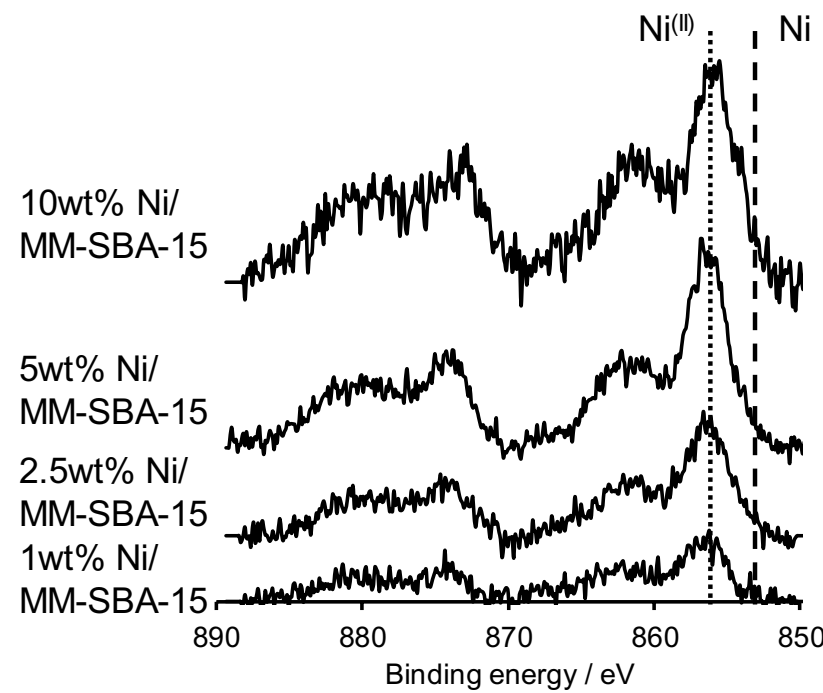

Fig. 2 Stacked Ni 2p XPS spectra offset for clarity, with binding energies of $\mathrm{Ni}$ metal and $\mathrm{NiO}$ indicated technique [35]. Average Ni particle sizes range from 1.4 to $3.0 \mathrm{~nm}$ for 1 and $10 \mathrm{wt} \%$ loading respectively, with all sample showing reasonably monomodal size distributions (Fig. 4b, d, f, and h). These sizes are considerably smaller than the average mesopore diameters, consistent with our study employing the ethyl glycol assisted impregnation for mesoporous only supports [23], and differ to non-assisted impregnated Ni/SBA-15 [15, 36]. Comparing our hierarchal MM-SBA-15 to the conventional SBA-15, we see a significant enhancement in $\mathrm{Ni}$ dispersion for the macroporous mesoporous framework. This decrease in average Ni nanoparticle size opens up more sites for catalysis, representing a more efficient utilisation of the support's available surface area.

The performance of our Ni/MM-SBA-15 catalyst series for hydrogen production, from ESR, was evaluated as a potential route for sustainable production [37]. $\mathrm{H}_{2}$ production was evaluated at $600{ }^{\circ} \mathrm{C}$, identical conditions to those used in our previous studies, to quantify initial activity and on-stream deactivation over the initial $2 \mathrm{~h}$ of the reaction (Fig. 5).
Fig. 3 Bright field STEM of MM-SBA-15; a macropore structure, $\mathbf{b}$ polystyrene macropore hard template, $\mathbf{c}$ mesopore channels, and $\mathbf{d}$ hexagonal packing of mesopores
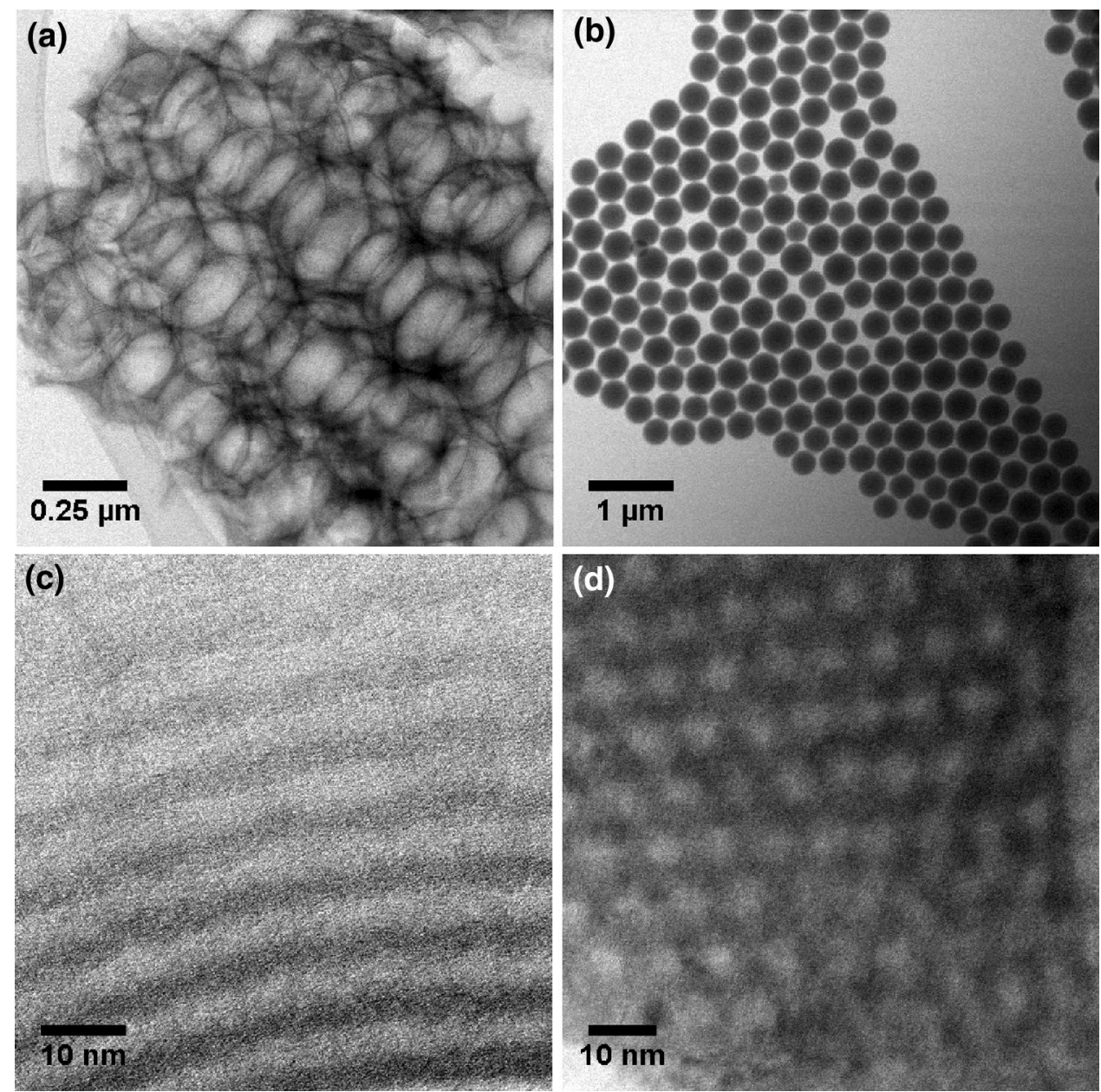
Fig. 4 HAADF-STEM and Ni particle size distribution of $\mathbf{a}$, b $10 \mathrm{wt} \% \mathrm{Ni} / \mathrm{MM}-\mathrm{SBA}-15$, c, d $5 \mathrm{wt} \% \mathrm{Ni} / \mathrm{MM}-\mathrm{SBA}-15$, e, f $2.5 \mathrm{wt} \% \mathrm{Ni} / \mathrm{MM}-\mathrm{SBA}-15, \mathbf{g}, \mathbf{h} 1$ wt $\%$ Ni/MM-SBA-15
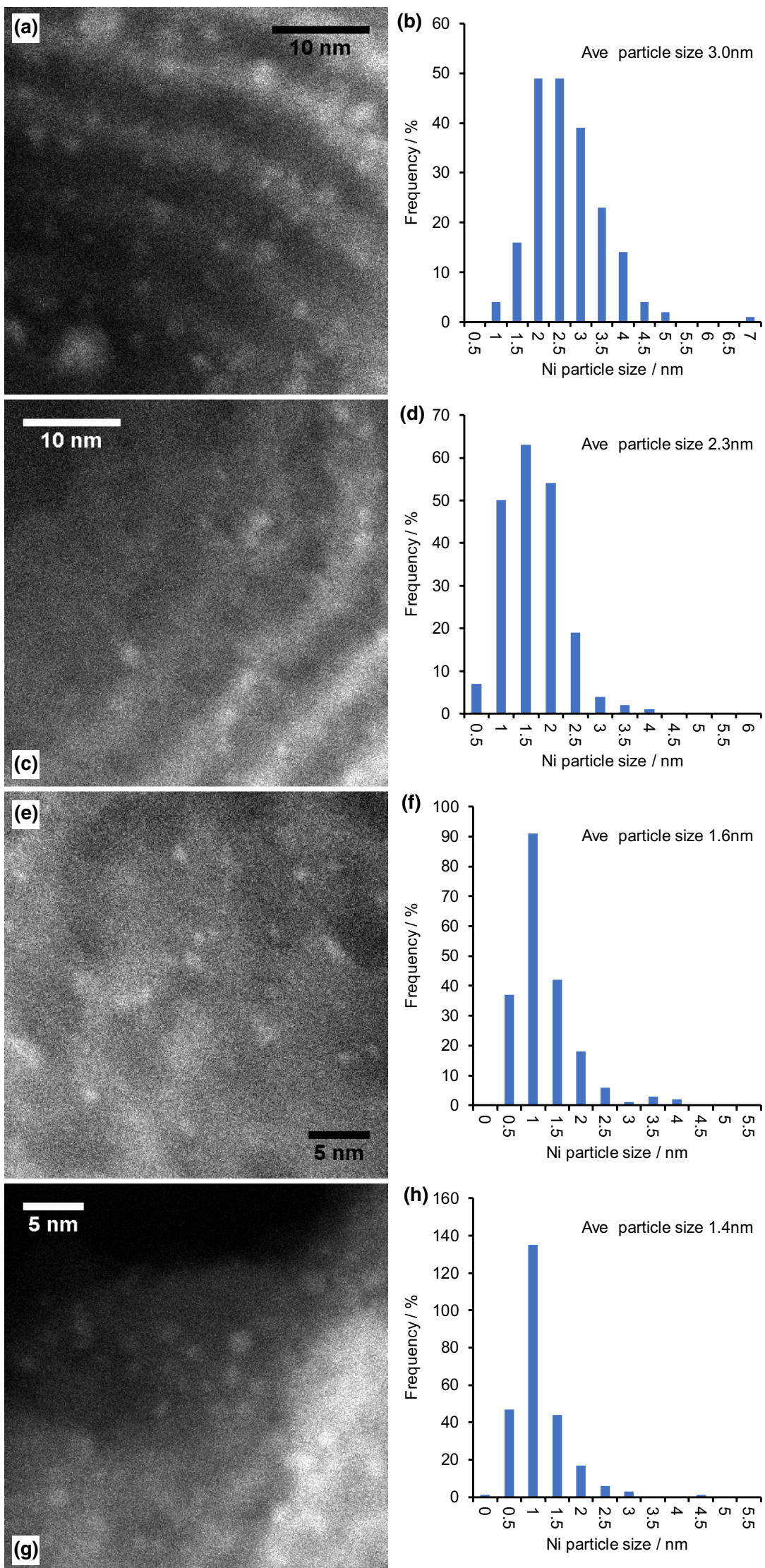

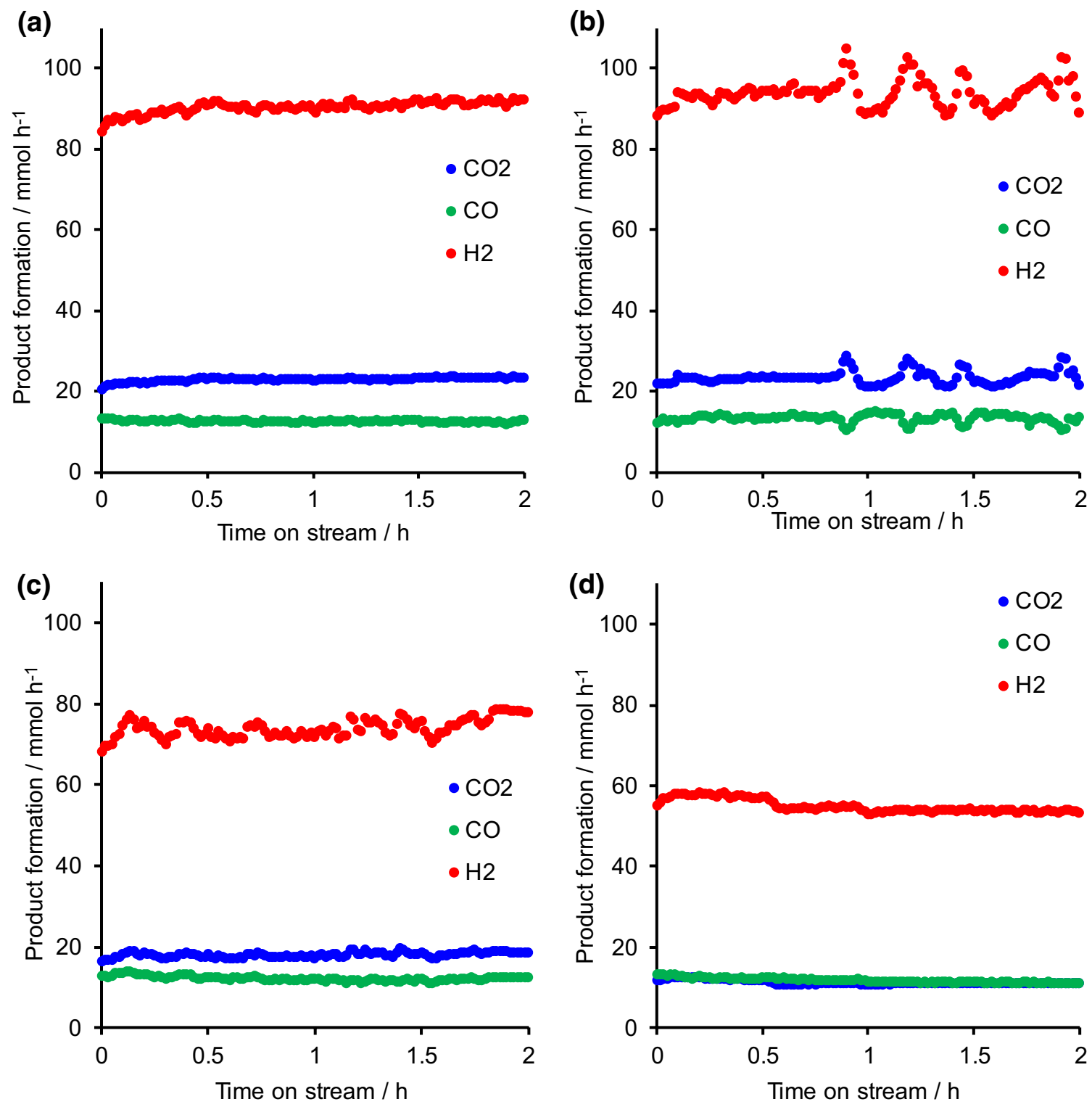

Fig. 5 Hydrogen production from ESR over a 10 wt $\%$ Ni/MM-SBA-15, b 5 wt $\%$ Ni/MM-SBA-15, c 2.5 wt $\%$ Ni/MM-SBA-15, and d 1 wt $\%$ Ni/ MM-SBA-15

This relatively short on-stream time frame represents the period over which considerable losses in performance were previously observed for conventional SBA-15, with up to a $50 \%$ decrease observed. The time on-stream profiles show unvarying hydrogen production across all four catalysts, with overall hydrogen production increasing with metal loading. This absence in observed deactivation reflects the highly beneficial role of macropore incorporation for this gas-phase reaction. The capacity of macroporosity to overcome coking (the deactivation route occurrent in conventional SBA15 ) is particularly striking given the relative dimensions of substrate and products $(0.38 \mathrm{~nm})$ and mesopore $(5.5 \mathrm{~nm})$ respectively. Furthermore, we can rule out a difference in on-stream stability to be a size effect, direct comparison of equal Ni particles sizes (10 wt $\%$ Ni/MM-SBA- 15 vs $2.5 \mathrm{wt} \%$ $\mathrm{Ni} / \mathrm{SBA}-15$ ) show deactivations of $\sim 0 \%$ vs $50 \%$ respectively. This also discredits differences in other physicochemical properties of the Ni sites being responsible, as these can be assumed to be comparable given the identical synthesis protocols and equal mesopore structure. Given that 90\% of the surface area in MM-SBA-15 is within the mesopores [38], the vast majority of the $\mathrm{Ni}$ can be expected to reside within these. Therefore, we conclude that the beneficial role of the macropores in preventing deactivation is diffusion driven, with the shortened mesopore domains resulting in a decrease in residence time of the substrate/products and 

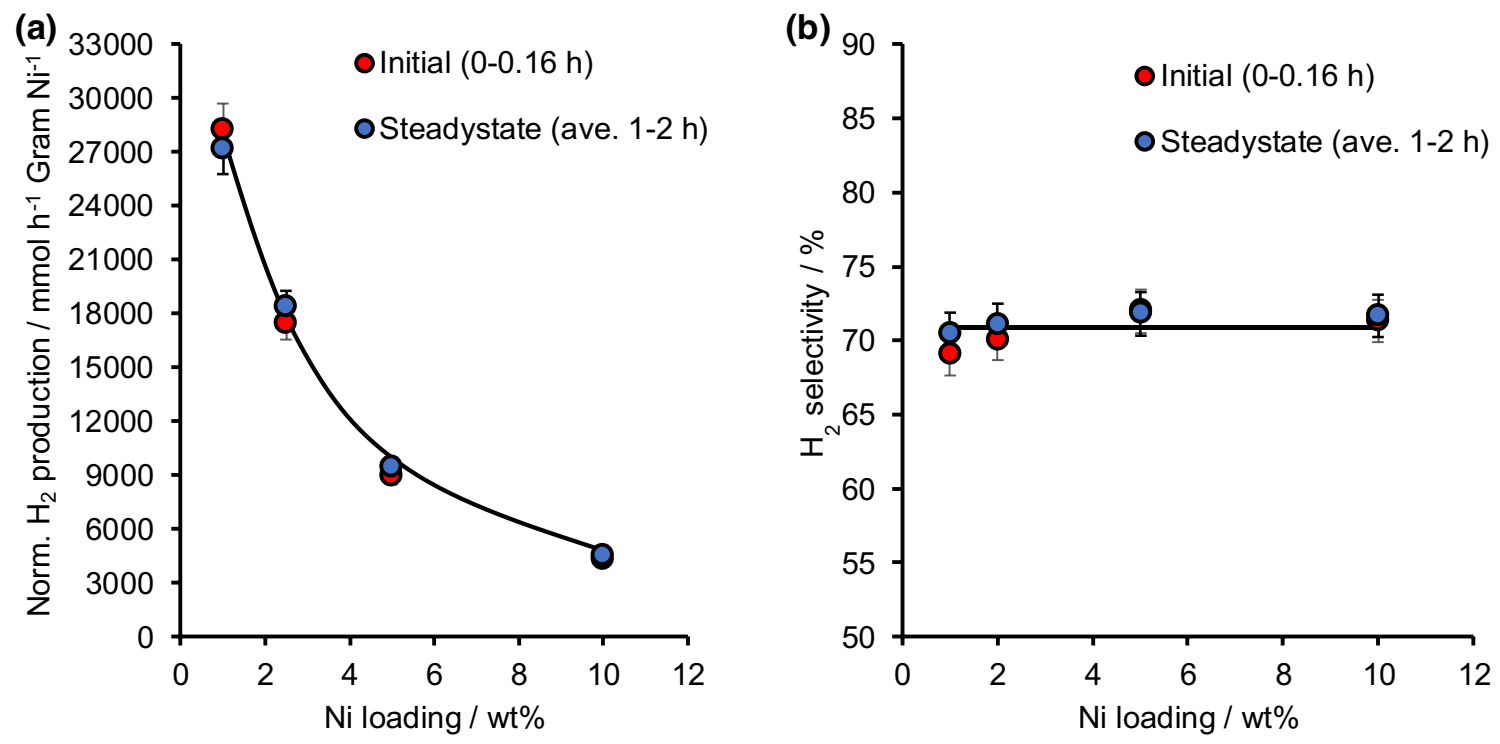

Fig. 6 a mass normalised initial and steady-state productivity of Ni/MM-SBA-15 and b initial and steady-state hydrogen selectivity of Ni/MMSBA-15

thus a decrease in potential for coke formation [39]. It is also worth noting that under our reaction conditions, ethanol conversion is not equilibrium limited, confirmed by simulation and in agreement with the literature [40, 41]. Therefore, an optimal ethanol conversion of $65 \%$ (5 wt $\%$ $\mathrm{Ni} / \mathrm{MM}-\mathrm{SBA}-15$ ) validates that our reaction conditions are reagent limited [27].

Mass normalised $\mathrm{H}_{2}$ productivity averaged over the initial $10 \mathrm{~min}$ of the reaction revealed an inverse correlation with loading (Fig. 6a). This MM-SBA-15 series shows enhancement in performance of up to $80 \%$, and an average across the series of $60 \%$ (for comparable loading), when benchmarked against our previously reported conventional SBA-15 [23]. The performance of these materials is also considerably greater than citric acid assisted sol-gel $\mathrm{Ni} / \mathrm{SiO}_{2}$ and $\mathrm{Ni} /$ SBA-15 prepared via aqueous incipient wetness impregnation (1375 and $850 \mathrm{mmol} \mathrm{h}^{-1} \mathrm{~g}_{\mathrm{Ni}}^{-1}$ respectively) $[15,19]$. The superior activity arises from a smaller Ni particle size, from higher metal dispersions, which is likely to result in the formation of unique ensembles which presumably favour a higher density of low coordination sites and is consistent with a size sensitive active species, as previously proposed. Quantitative comparison of these initial activities and steady-state activities confirms the absence of deactivation.

As observed previously for our Ni/SBA-15 and Ni/KIT-6 catalyst families, the Ni/MM-SBA-15 series displays a common hydrogen selectivity of $70 \%$ (Fig. 6b), with CO and $\mathrm{CO}_{2}$ formation in approximately a 1:1 ratio. This common selectivity across this series (and our previous investigation) [23] is indicative of a common reforming mechanism that is independent of nickel particle size, support, and also time on stream when deactivation is absent.

\section{Conclusions}

The incorporation of secondary macropore networks within mesoporous SBA-15, via a hard-templating strategy, yields an excellent catalyst support framework for ESR. Highly dispersed Ni nanoparticles within our MM-SBA-15 skeleton show excellent activity and selectivity for hydrogen production. The open architecture for the bimodal porous support material enables superior $\mathrm{Ni}$ dispersion during catalyst preparation, which subsequently imparts improved performance, both initially and under steady-state conditions, with the latter due to support resistance to on-stream deactivation.

Acknowledgements XPS data analysis was performed at the EPSRC National Facility for XPS ('HarwellXPS'), operated by Cardiff University and UCL, under contract No. PR16195. The authors declare that they have no conflict of interest.

Open Access This article is licensed under a Creative Commons Attribution 4.0 International License, which permits use, sharing, adaptation, distribution and reproduction in any medium or format, as long as you give appropriate credit to the original author(s) and the source, provide a link to the Creative Commons licence, and indicate if changes were made. The images or other third party material in this article are included in the article's Creative Commons licence, unless indicated otherwise in a credit line to the material. If material is not included in the article's Creative Commons licence and your intended use is not permitted by statutory regulation or exceeds the permitted use, you will 
need to obtain permission directly from the copyright holder. To view a copy of this licence, visit http://creativecommons.org/licenses/by/4.0/.

\section{References}

1. Huber GW, Corma A (2007) Synergies between bio- and oil refineries for the production of fuels from biomass. Angew Chem Int Ed 46(38):7184-7201

2. Galvez-Martos J-L et al (2018) Conceptual design of a $\mathrm{CO}_{2}$ capture and utilisation process based on calcium and magnesium rich brines. J Carbon Dioxide Util 27:161-169

3. Ni M, Leung DYC, Leung MKH (2007) A review on reforming bio-ethanol for hydrogen production. Int J Hydrogen Energy 32(15):3238-3247

4. Guczi L, Erdôhelyi A (2012) Catalysis for alternative energy generation. Springer, New York

5. Sehested J (2006) Four challenges for nickel steam-reforming catalysts. Catal Today 111(1-2):103-110

6. Phung TK et al (2020) Effect of supports and promoters on the performance of ni-based catalysts in ethanol steam reforming. Chem Eng Technol 43(4):672-688

7. Mattos LV et al (2012) Production of hydrogen from ethanol: review of reaction mechanism and catalyst deactivation. Chem Rev 112(7):4094-4123

8. Dang CX et al (2020) Mg-promoted Ni-CaO microsphere as bi-functional catalyst for hydrogen production from sorptionenhanced steam reforming of glycerol. Chem Eng J 383:9

9. Ghungrud SA, Vaidya PD (2020) Improved hydrogen production from sorption-enhanced steam reforming of ethanol (SESRE) using multifunctional materials of cobalt catalyst and $\mathrm{Mg}-, \mathrm{Ce}-$, and $\mathrm{Zr}$-modified $\mathrm{CaO}$ sorbents. Ind Eng Chem Res 59(2):693-703

10. Lu JC et al (2020) Weakening the metal-support strong interaction to enhance catalytic performances of alumina supported Ni-based catalysts for producing hydrogen. Appl Catal B 263:13

11. Zhang ZM et al (2020) Steam reforming of acetic acid over Ni-Ba/ $\mathrm{Al}_{2} \mathrm{O}_{3}$ catalysts: impacts of barium addition on coking behaviors and formation of reaction intermediates. J Energy Chem 43:208-219

12. Li S, Gong J (2014) Strategies for improving the performance and stability of Ni-based catalysts for reforming reactions. Chem Soc Rev 43(21):7245-7256

13. Greluk M, Rotko M, Turczyniak-Surdacka S (2020) Comparison of catalytic performance and coking resistant behaviors of cobaltand nickel based catalyst with different $\mathrm{Co} / \mathrm{Ce}$ and $\mathrm{Ni} / \mathrm{Ce}$ molar ratio under SRE conditions. Appl Catal A 590:17

14. Li D et al (2015) Ceria-promoted Ni/SBA-15 catalysts for ethanol steam reforming with enhanced activity and resistance to deactivation. Appl Catal B 176-177:532-541

15. Rossetti I et al (2014) Silica and zirconia supported catalysts for the low-temperature ethanol steam reforming. Appl Catal B 150:257-267

16. Albarazi A, Galvez ME, Da Costa P (2015) Synthesis strategies of ceria-zirconia doped Ni/SBA-15 catalysts for methane dry reforming. Catal Commun 59:108-112

17. Osatiashtiani A et al (2015) Hydrothermally stable, conformal, sulfated zirconia monolayer catalysts for glucose conversion to 5-HMF. ACS Catal 5(7):4345-4352

18. Wu C, Williams PT (2010) A novel nano-Ni/SiO 2 catalyst for hydrogen production from steam reforming of ethanol. Environ Sci Technol 44(15):5993-5998

19. Wu C, Williams PT (2011) Hydrogen production from steam reforming of ethanol with nano- $\mathrm{Ni} / \mathrm{SiO}_{2}$ catalysts prepared at different Ni to citric acid ratios using a sol-gel method. Appl Catal B 102(1-2):251-259

20. Parlett CMA et al (2013) Mesoporous silicas as versatile supports to tune the palladium-catalyzed selective aerobic oxidation of allylic alcohols. ChemCatChem 5(4):939-950

21. He SY et al (2015) Hydrogen production by ethanol steam reforming over Ni/SBA-15 mesoporous catalysts: effect of Au addition. Catal Today 258:162-168

22. Vizcaino AJ, Carrero A, Calles JA (2016) Comparison of ethanol steam reforming using Co and Ni catalysts supported on SBA-15 modified by Ca and Mg. Fuel Process Technol 146:99-109

23. Parlett CMA et al (2017) Tailored mesoporous silica supports for $\mathrm{Ni}$ catalysed hydrogen production from ethanol steam reforming. Catal Commun 91:76-79

24. Villagran-Olivares AC et al (2020) Effect of EDTA in preparation of Ni catalysts toward a carbon-resistant ethanol reforming. Appl Catal B 264:8

25. Parlett CMA et al (2013) Hierarchically ordered nanoporous Pd/ SBA-15 catalyst for the aerobic selective oxidation of sterically challenging allylic alcohols. ACS Catal 3(9):2122-2129

26. Dhainaut J et al (2010) Hierarchical macroporous-mesoporous SBA-15 sulfonic acid catalysts for biodiesel synthesis. Green Chem 12(2):296-303

27. Schuth F, Ward MD, Buriak JM (2018) Common pitfalls of catalysis manuscripts submitted to chemistry of materials. Chem Mater 30(11):3599-3600

28. Montero $\mathrm{C}$ et al (2018) Optimum operating conditions in ethanol steam reforming over a Ni/La $\mathrm{O}_{3}$-alpha $\mathrm{Al}_{2} \mathrm{O}_{3}$ catalyst in a fluidized bed reactor. Fuel Process Technol 169:207-216

29. Yan J-M et al (2009) Synthesis of longtime water/air-stable ni nanoparticles and their high catalytic activity for hydrolysis of ammonia-borane for hydrogen generation. Inorg Chem 48(15):7389-7393

30. Galarneau A et al (2001) True microporosity and surface area of mesoporous SBA-15 silicas as a function of synthesis temperature. Langmuir 17(26):8328-8335

31. Kruk M et al (2000) Characterization of the porous structure of SBA-15. Chem Mater 12(7):1961-1968

32. Zhao DY et al (2000) Morphological control of highly ordered mesoporous silica SBA-15. Chem Mater 12(2):275

33. Isaacs MA et al (2019) Unravelling mass transport in hierarchically porous catalysts. J Mater Chem A 7(19):11814-11825

34. Zhao DY et al (1998) Nonionic triblock and star diblock copolymer and oligomeric surfactant syntheses of highly ordered, hydrothermally stable, mesoporous silica structures. J Am Chem Soc 120(24):6024-6036

35. Pennycook SJ, Boatner LA (1988) Chemically sensitive structure-imaging with a scanning-transmission electron-microscope. Nature 336(6199):565-567

36. Calles JA et al (2014) Hydrogen production by glycerol steam reforming over SBA-15-supported nickel catalysts: effect of alkaline earth promoters on activity and stability. Catal Today 227:198-206

37. Dunn S (2002) Hydrogen futures: toward a sustainable energy system. Int J Hydrogen Energy 27(3):235-264

38. Parlett CMA et al (2016) Spatially orthogonal chemical functionalization of a hierarchical pore network for catalytic cascade reactions. Nat Mater 15(2):178

39. Weissenberger T et al (2019) Hierarchical MFI type zeolites with intracrystalline macropores: the effect of the macropore size on the deactivation behaviour in the MTO reaction. Catal Sci Technol 9(12):3259-3269

40. Wang W, Wang YQ (2008) Thermodynamic analysis of steam reforming of ethanol for hydrogen generation. Int J Energy Res 32(15):1432-1443 
41. Tahir $\mathrm{M}$ et al (2017) Thermodynamic and experimental analysis on ethanol steam reforming for hydrogen production over $\mathrm{Ni}$ modified TiO2/MMT nanoclay catalyst. Energy Convers Manag $154: 25-37$
Publisher's Note Springer Nature remains neutral with regard to jurisdictional claims in published maps and institutional affiliations. 